\title{
On Optical Characterization of Carrier Lifetimes in GaN Layers by Time-Resolved Four-Wave Mixing and Photoluminescence Techniques
}

\author{
T. Malinauskas* AND K. JARAŠIŪNAS \\ Department of Semiconductor Optoelectronics
}

Institute of Materials Science and Applied Research

Vilnius University, Sauletekio ave. 9 bld. 3, 10222 Vilnius, Lithuania

We provide numerical analysis of nonequilibrium carrier dynamics in GaN layers at interband photoexcitation by a picosecond light pulse. By solving the continuity equation for bipolar carrier plasma, we analyze spatial and temporal evolution of carrier density. We show that fast carrier diffusion to the bulk determines the carrier in-depth profile in GaN epilayers with a thickness larger than the carrier diffusion length. By integrating the carrier spatial profiles at experimental conditions, corresponding to time-resolved four-wave mixing and time-resolved photoluminescense we simulate the four-wave mixing and time-resolved photoluminescense kinetics in subnanosecond time domain. The modeling data using parameters of the studied GaN epilayers (their thickness, diffusion coefficient, carrier lifetime, and absorption coefficients at emission wavelengths) were compared with the experimental results. The analysis provided conditions at which the discrepancy between the measured carrier lifetime by time-resolved photoluminescense and time-resolved four-wave mixing may occur. For hydride-vapor phase epitaxy GaN layers with a large diffusion length, the fast photoluminescense kinetics are confirmed by modeling and experiments that they are due to diffusion governed carrier in-depth redistribution, while four-wave mixing kinetics remain insensitive for carrier in-depth redistribution.

PACS numbers: $72.20 . \mathrm{Jv}, 78.47 .+\mathrm{p}, 78.55 . \mathrm{Cr}$

\section{Introduction}

Time-resolved optical characterization is a versatile tool for studying the features of recombination and transport processes in highly excited semiconductors

*corresponding author; e-mail: tadas.malinauskas@ff.vu.lt 
and determination of related parameters, such as carrier lifetimes and diffusion coefficients. Time-resolved photoluminescence (TRPL) spectroscopy technique is commonly used to study spontaneous and stimulated emission processes in nitride materials for light-emitting devices, while its applicability is dependent on efficiency of radiative emission. Time-resolved four-wave mixing (TR FWM) on free carrier gratings is a technique able to control spatial and temporal nonequilibrium carrier redistribution and is equally sensitive to mechanisms of radiative and nonradiative recombination; in addition, it provides carrier diffusion parameters. Recently, both techniques have been applied for complementary studies of carrier dynamics in differently grown GaN samples [1,2]. Comparison of the determined carrier recombination times has shown only satisfactory agreement for high GaN layers and thus required more explicit analysis of the nonequilibrium processes in carrier plasma with very nonhomogeneous in-depth profile. In this paper, we provide numerical analysis of carrier dynamics in a few ns time domain after photoexcitation and simulate the carrier lifetimes, determined by TRPL and FWM techniques.

\section{Experimental conditions}

The experiments were carried out on two differently grown GaN samples. The $270 \mu \mathrm{m}$ thick free-standing layer was grown by hydride-vapor phase epitaxy (HVPE) on a two-step epitaxial lateral overgrown (2S-ELO) GaN template on sapphire [3]. The $1.7 \mu \mathrm{m}$ thick nominally undoped GaN layer was grown by a standard metalorganic chemical vapor deposition (MOCVD) technique on sapphire [2].

Carrier dynamics in GaN samples was investigated using the non-degenerate FWM technique and TRPL techniques. For FWM measurements, interference pattern of two 25 ps duration laser beams at $355 \mathrm{~nm}$ wavelength $(h \nu=3.49 \mathrm{eV})$ created a modulated free-carriers distribution $N(x)=N_{0}(1+\cos (2 \pi x / \Lambda))$ with period $\Lambda$. In turn, the refractive index of a material was changed in the interference peaks $(\Delta n \propto \Delta N)$ and created a dynamic diffraction grating. The grating was probed by weakly absorbed beam at $1064 \mathrm{~nm}$ which propagated through the sample and diffracted on the free-carrier grating. The TRPL was studied under excitation at $266 \mathrm{~nm}$ wavelength (using a pulse duration of $25 \mathrm{ps}$ and spot size of $1 \mathrm{~mm}$ ) and collected in a backward geometry, using a $0.4 \mathrm{~m}$ grating monochromator [1]. Temporal resolution of $\sim 20$ ps was achieved using an optical Kerr shutter. Bandto-band spontaneous PL transients were measured at $3.4 \mathrm{eV}$.

For both the techniques used, the exciting light was absorbed in $\alpha^{-1} \sim$ $100 \mathrm{~nm}$ thin surface layer of GaN, creating the bipolar carrier plasma with density of about $10^{19} \mathrm{~cm}^{-3}$. Carrier diffusion from surface to the bulk increased the excited layer thickness up to $1-2 \mu \mathrm{m}$ in time domain of measurements. Since the probe beam in FWM technique integrates the plasma modulation over the sample depth, this makes the diffraction signal dependent on the total number of photogenerated electron-hole pairs in the sample and not on a specific density 
profile. In case of PL, the emission is quite strongly absorbed in the sample $\left(\alpha_{\mathrm{PL}} \sim(5-10) \times 10^{3} \mathrm{~cm}^{-1}[2]\right)$, thus the measured PL signal corresponds to radiative recombination of carriers, present in a surface layer of limited thickness.

\section{Numerical modeling}

Nonequilibrium carrier dynamics in photoexcited semiconductor crystal is well described by one-dimensional model, if the carrier in-depth distribution is homogeneous (i.e. at weak excitation absorption or if a sample thickness is less than the carrier diffusion length). However, under the strong absorption in relatively thick layer, the fast changes of carrier in-depth profile take place due to carrier diffusion to the bulk. Therefore, a two-dimensional model has to be employed [4], which allows modeling of spatial and temporal carrier redistribution after their generation by a short laser pulse. According to this model, the two-dimensional carrier density $N(x, z, t)$ in a layer of a finite thickness $d$ is described by the following equation:

$$
\begin{aligned}
& \frac{\partial N(x, z, t)}{\partial t}= \\
& \quad \nabla[D(N) \nabla N(x, z, t)]-\frac{N(x, z, t)}{\tau_{R}}-B N^{2}(x, z, t)+G(x, z, t)
\end{aligned}
$$

with boundary conditions

$$
\left.\frac{\partial N(x, z, t)}{\partial z}\right|_{z=0}=\frac{S_{0}}{D(N)} N(x, 0, t),\left.\quad \frac{\partial N(x, z, t)}{\partial z}\right|_{z=d}=\frac{S_{d}}{D(N)} N(x, d, t)
$$

where $B$ is the bimolecular recombination rate, and $S_{0}$ and $S_{d}$ are surface recombination velocities at the front and back surface, correspondingly. The instantaneous carrier distribution depends on several parameters, particularly on $D, \tau_{R}, S$, and absorption coefficient $\alpha$. The model takes into account both linear and nonlinear carrier recombination and diffusion. The solution of (1) provides the spatio-temporal distribution of carriers $N(x, z, t)$, which is used to calculate the refractive index modulation $\Delta n$ and diffraction efficiency $\eta_{\mathrm{FWM}}$ :

$$
\eta_{\mathrm{FWM}}(t) \propto\left[\int_{0}^{d} \Delta N(z, t) \mathrm{d} z\right]^{2} .
$$

Correspondingly, the carrier profiles are used to calculate the instantaneous PL intensity:

$$
I_{\mathrm{PL}}(t) \propto \int_{0}^{d} \Delta N^{2}(z, t) \exp \left(-\alpha_{\mathrm{PL}} z\right) \mathrm{d} z .
$$

We note that both FWM and PL depend nonlinearly on carrier density, but in a different way: the diffraction depends on the probe beam phase shift, $\Phi=$ $(2 \pi \Delta n d) / \lambda$, which is integrated during the beam propagation through the sample, and the total accumulated value in optical path change, $(\Delta n \cdot d)^{2}$, determines 
the diffracted beam efficiency, $\eta_{\mathrm{FWM}} \propto \Phi^{2}$. As a rule, the probe beam is weakly absorbed in the crystal to ensure the high intensity of diffraction signal. Moreover, normalization of the diffracted beam to transmitted one is used for determination of the diffraction efficiency, $\eta_{\mathrm{FWM}}=I_{\mathrm{diffr}} / I_{\text {trans }}$, that makes the $\eta_{\mathrm{FWM}}$ value insensitive to probe beam absorption even at its presence.

The PL signal is generated in a sample locally and nonlinearly, $I_{\mathrm{PL}} \propto N^{2}$, and may be partially or strongly reabsorbed until it propagates through the sample. Therefore, in a general case, only the limited thickness of a photoexcited layer may contribute to PL signal, and all three factors: the carrier spatial redistribution, their density, and the reabsorption effects, must be explicitly considered when the photoelectric parameters of a semiconductor are needed to be determined.

\section{Results and discussions}

Correct numerical modeling of carrier dynamics requires using photoelectric parameters of the crystals used for the given experiments. Therefore we applied TR FWM technique to extract the values of diffusion coefficient $D$ and carrier lifetime $\tau_{R}$ in the HVPE sample with low dislocation density. In Fig. 1a, the measured grating decay kinetics at various grating periods are plotted, and the faster decay at smaller grating period points out the contribution of carrier diffusion along the
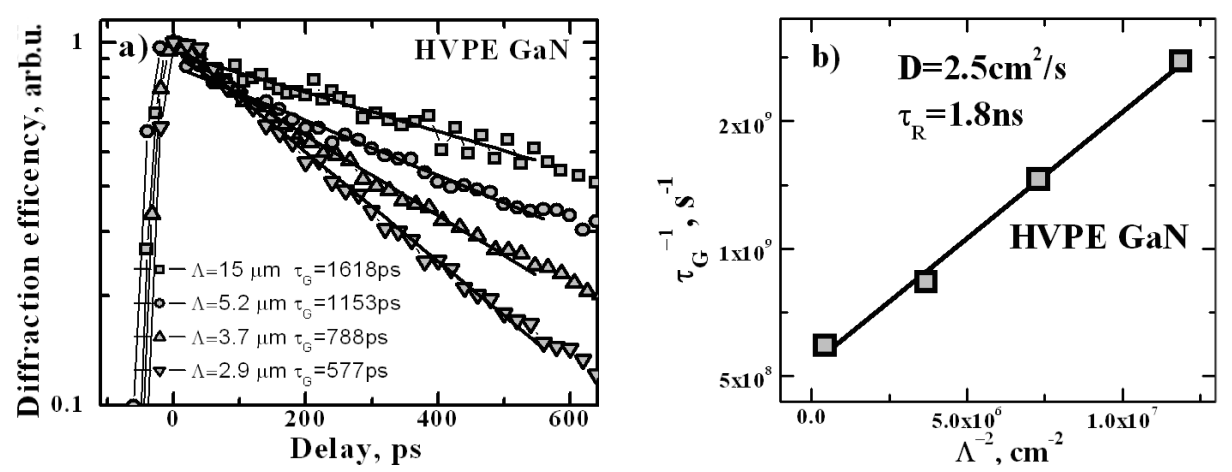

Fig. 1. Grating decay kinetics at various periods in HVPE GaN sample (a) and the determined values of bipolar diffusion coefficient $D$ and carrier lifetime $\tau_{R}$ (b).

grating vector. The plot of inverse grating decay time vs. its period (Fig. 1b) provides the $D=2.5 \mathrm{~cm}^{2} / \mathrm{s}$ and $\tau_{R}=1.8 \mathrm{~ns}$ values in thick HVPE sample, according to the relationship $1 / \tau_{G}=1 / \tau_{R}+1 / \tau_{D}$, where $\tau_{D}=\Lambda^{2} / 4 \pi^{2} D$ is the diffusive grating decay time. The $D=1.4 \mathrm{~cm}^{2} / \mathrm{s}$ and $\tau_{R}=0.46 \mathrm{~ns}$ values for the standard MOCVD sample were measured in a similar way.

In Fig. 2 we present the carrier spatial redistribution in $270 \mu \mathrm{m}$ thick HVPE layer after excitation by picosecond laser pulse. Carrier diffusion results in enhanced effective thickness of the photoexcited region at the surface. The role of 
surface recombination is negligible for the used value $S=10^{4} \mathrm{~cm} / \mathrm{s}$, while for $S>5 \times 10^{4} \mathrm{~cm} / \mathrm{s}$ it would diminish the carrier density as well [5]. Carrier bimolecular recombination is not included in the modeling, since diffraction kinetics are purely monoexponential (in measured timescale), i.e. carrier recombination is mainly governed by linear recombination. The carrier density at the surface is governed by carrier diffusion: at $\Delta t=500 \mathrm{ps}$, the diffusion leads to reduction of carrier density by 5 times instead of expected factor of 1.2 solely due to recombination (i.e. $1 / \exp (-500 / 3000)=1.2)$.

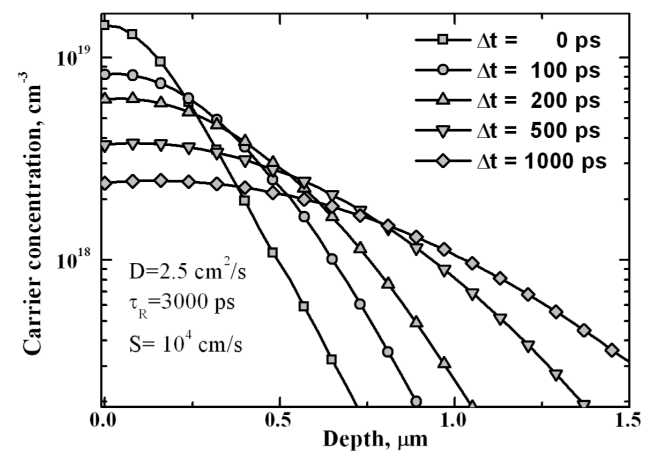

Fig. 2. Evolution of carrier spatial in-depth profile after photoexcitation.

The modeling of FWM and PL kinetics disclose their sensitivity to carrier redistribution due to diffusion (Fig. 3a). If the diffusion is not effective during the measurement time, then the FWM and PL kinetics become nearly the same. However, at the $D$ value as low as $0.25 \mathrm{~cm}^{2} / \mathrm{s}$ (and low carrier recombination rate, e.g. $\tau_{R}=4 \mathrm{~ns}$ ) the carrier redistribution is noticeable in PL kinetics, as the nonlinear origin of PL signal quadratically enhances the decreased carrier density in the surface region. In contrary, FWM efficiency remains insensitive to carrier in-depth profile. The role of recombination (Fig. 3b) competes with diffusion and thus can be reliably seen if the average carrier recombination rate is high enough
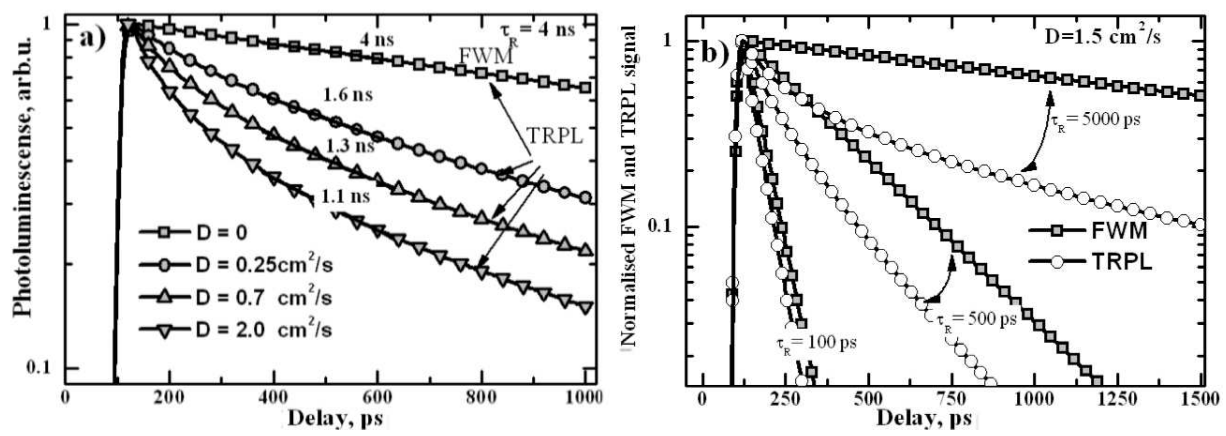

Fig. 3. Role of carrier diffusion (a) and recombination (b) in the PL and FWM kinetics. 
and exceeds the instantaneous changes in carrier profile due to diffusion. The discrepancy between the carrier lifetime values determined by TRPL and FWM is larger for samples with higher diffusion coefficients and longer recombination times, i.e. in case of large carrier diffusion length.

The modeling of carrier dynamics for the experimentally studied GaN crystals has been carried out to verify the predicted peculiarities due to carrier diffusion. In Fig. 4 we compare the measured and calculated FWM and PL kinetics. PL kinetics are faster than the FWM ones because of two factors: (i) the carriers
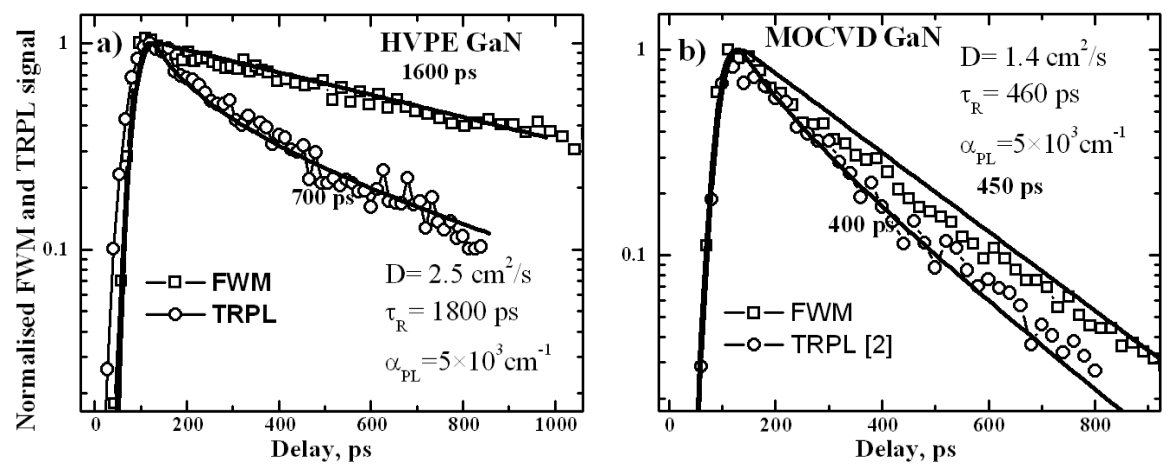

Fig. 4. Comparison of the calculated curves (lines) and experimental data (points) of TRPL and TR FWM kinetics in HVPE GaN (a), and in standard MOCVD GaN (b) samples.

escape from the area which contributes to the PL signal and (ii) the quadratic relationship between the PL signal and carrier density $\left(I_{\mathrm{PL}} \propto N^{2}\right)$ enhances the carrier density reduction effect caused by the diffusion. We note that these factors introduce a systematic factor in the measurable PL times, which may differ a few times from a real carrier lifetime.

\section{Conclusions}

The numerical analysis of two-dimensional carrier dynamics in GaN epilayers has been performed and the data compared with the experimental PL and FWM kinetics. It was shown that the diffusion-caused carrier in-depth redistribution during the measurement time (up to $1 \mathrm{~ns}$ ) is responsible for the observed discrepancy between the experimental PL and FWM kinetics. On the other hand, the techniques are complementary and can be used for more detailed studies of competition of radiative/nonradiative channels of carrier recombination as well for the role of diffusion processes. 


\section{Acknowledgment}

The work is supported by European Commission Contract No. G5MA-CT-2002-04047, NATO's Scientific Affairs Division (Science for Peace Programme Project SfP-974476), and Lithuanian Education and Science Foundation.

\section{References}

[1] K. Jarašiūnas, T. Malinauskas, A. Kadys, R. Aleksiejūnas, M. Sūdžius, S. Miasojedovas, S. Juršènas, A. Žukauskas, D. Gogova, A. Kakanakova-Georgieva, E. Janzén, H. Larsson, B. Monemar, P. Gibart, B. Beaumont, Phys. Status Solidi C 2, 1006 (2005).

[2] G.P. Yablonskii, A.L. Gurskii, V.N. Pavlovskii, E.V. Lutsenko,V.Z. Zubialevich, T.S. Shulga, A.I. Stognij, H. Kalisch, A. Szymakowski, R.H. Jansen, A. Alam, B. Schineller, M. Heuken, J. Cryst. Growth 275, e1733 (2005).

[3] D. Gogova, A. Kasic, H. Larsson, C. Hemmingsson, B. Monemar, F. Tuomisto, K. Saarinen, L. Dobos, B. Pecz, P. Gibart, B. Beaumont, J. Appl. Phys. 96, 799 (2004).

[4] C.A. Hoffman, K. Jarasiunas, H.J. Gerritsen, A.V. Nurmikko, Appl. Phys. Lett. 33, 536 (1978).

[5] K. Jarasiunas, R. Aleksiejunas, T. Malinauskas, V. Gudelis, M. Sudzius, A. Maasdorf, F. Brunner, M. Weyers, Eur. Phys. J. Appl. Phys. 27, 181 (2004). 